\title{
Dynamic object representations in infants with and without fragile $X$ syndrome
}

\author{
Faraz Farzin ${ }^{1,2}$ and Susan M. Rivera ${ }^{1,2,3 *}$ \\ 1 Department of Psychology, University of California Davis, Davis, CA, USA \\ 2 Center for Mind and Brain, University of California Davis, Davis, CA, USA \\ ${ }^{3}$ M.I.N.D. Institute, University of California Davis Medical Center, Sacramento, CA, USA
}

\section{Edited by:}

Elizabeth D. O'Hare,

University of California, USA

\section{Reviewed by:}

Gaia Scerif, University of Oxford, UK Melanie Palomares, Johns Hopkins

University, USA

\section{${ }^{*}$ Correspondence:}

Susan M. Rivera, Center for Mind and Brain, University of California Davis, 202 Cousteau Place, Suite 250, Davis, CA 95618, USA.

e-mail:srivera@ucdavis.edu
Our visual world is dynamic in nature. The ability to encode, mentally represent, and track an object's identity as it moves across time and space is critical for integrating and maintaining a complete and coherent view of the world. Here we investigated dynamic object processing in typically developing (TD) infants and infants with fragile $X$ syndrome (FXS), a single-gene disorder associated with deficits in dorsal stream functioning. We used the violation of expectation method to assess infants' visual response to expected versus unexpected outcomes following a brief dynamic (dorsal stream) or static (ventral stream) occlusion event. Consistent with previous reports of deficits in dorsal stream-mediated functioning in individuals with this disorder, these results reveal that, compared to mental age-matched TD infants, infants with FXS could maintain the identity of static, but not dynamic, object information during occlusion. These findings are the first to experimentally evaluate visual object processing skills in infants with FXS, and further support the hypothesis of dorsal stream difficulties in infants with this developmental disorder.

Keywords: object tracking, occlusion, motion, dorsal/ventral visual streams, attention

\section{INTRODUCTION}

Our visual world is dynamic in nature; we move and objects around us move. For example, an object can go out of sight as our eye or head position changes, or if moving, it can go out of sight when obstructed by other objects. Despite incomplete perceptual information during an object's occlusion, the adult visual system is able to form, maintain, and if necessary, track a mental representation of an object such that a coherent and complete visual scene is perceived across time and space. A critical question remains unanswered, however: to what extent do infants possess the ability to preserve an accurate representation of an object's identity during a dynamic occlusion event?

Previous studies have typically used the preferential looking paradigm to show that from a young age infants look longer at the outcome of a static occlusion event when a feature of the hidden object is changed compared to an event in which features remain the same. Extensive research using this paradigm has also provided evidence that the specific features which infants use to discriminate the identity of an object change across development. Infants as young as 4 months primarily represent occluded objects based on spatial information such as location and size (Baillargeon, 1993; Spelke et al., 1994; Mareschal and Johnson, 2003), and by the end of the first year infants use surface properties including shape ( 7 months), texture (11 months) and color (12 months) (Wilcox, 1999; Leslie and Kaldy, 2001; Wilcox and Schweinle, 2002; Kaldy and Leslie, 2003). Aspects of infants' perception, such as encoding and binding of object properties to build and maintain a representation during a static occlusion event, have been well characterized. Less is known about the ability of infants to maintain an object representation during a dynamic occlusion event, namely, whether infants can track a mental representation across time and space (Richardson and Kirkham, 2004).
The developmental hierarchy in the type of information that infants use to represent occluded objects closely maps on to the two distinct visual processing streams in the brain; the ventral and dorsal pathways. The occipito-temporal ventral stream involves processing of object features such as form and color, and projects to the inferotemporal cortex, while processing of information involved in guiding actions, including spatial location and motion, are served by the occipito-parietal dorsal stream, extending to the posterior parietal cortex (Ungerleider and Mishkin, 1982; Milner and Goodale, 1995). One account of this hierarchy proposes that infants initially set up an object representation based on spatial information (Leslie et al., 1998; Tremoulet et al., 2000; Leslie and Kaldy, 2001; Wilcox and Schweinle, 2002; Kaldy and Leslie, 2003) because, though the ventral and dorsal pathways are each operative early in development, information that is carried by each pathway remains segregated until the end of the first year of life, with the dorsal stream developing first but also thought to have a longer maturational time course (Atkinson, 1993; Leslie et al., 1998; Johnson et al., 2001; Kaldy and Leslie, 2003; Mareschal and Johnson, 2003). It has therefore been proposed that during the early months of development, performance on tasks requiring predominantly single stream processing may be superior to performance on tasks requiring integration of information across the two streams. Once the connections between the two pathways become functionally mature, infants are able to keep track of both an object's location in space (dorsal) and its featural properties (ventral), in the service of identifying an object as changed or unchanged following an occlusion event. Indeed, most everyday activities are guided by extensive interaction between dorsal and ventral representations in order to integrate features required for planning and executing actions. 
The functional, anatomical, and developmental dissociation between ventral and dorsal pathways has been influential for researchers investigating visual processing in various developmental disorders. Because of its protracted developmental time course, it has been suggested that the dorsal stream is particularly vulnerable to atypical development (Atkinson, 2000), thereby leading to a preponderance of dorsal stream deficits in developmental disorders, including fragile X syndrome (FXS).

FXS is the most is the most prevalent form of inherited mental retardation, with 1 in 3,800 males estimated to have the FXS full mutation and as many as 1 in 2,300 women estimated to carry the full mutation on at least one X chromosome (Crawford et al., 2001; Beckett et al., 2005). FXS is also the most common single-gene cause of autism (Reddy, 2005). The neurodevelopmental disorder is caused by the silencing of a single gene on the X chromosome, the Fragile X Mental Retardation 1 gene (Verkerk et al., 1991), which results in the reduction or absence of the fragile $\mathrm{X}$ mental retardation protein (FMRP) coded for by the gene. FMRP plays a key role in the post-synaptic development of dendritic spine morphology and acts as a repressor by regulating the translation of multiple dendritic mRNAs involved in synaptic development and function (Brown et al., 1982; Irwin et al., 2002). Dendritic abnormalities have been found in occipito-parietal areas of FMR1 knock-out mice as well as visual cortices of autopsied tissue from patients with FXS (Comery et al., 1997; Irwin et al., 2002), suggesting that FMRP is an important protein in the development of neural networks involving visual areas of the brain. Because of its specific single gene etiology, FXS offers a unique opportunity to examine the functional role of a specific gene product on neurocognitive development, and more specifically on functions supported by the dorsal stream.

There is ample evidence showing that visual processing deficits observed in individuals with FXS are not global in nature; rather, deficits in this population are specific to tasks mediated by the dorsal stream. A finding that emerges consistently across neuropsychological studies in children and adults with FXS is that affected individuals perform worse than typically developing (TD) controls on tasks requiring visual-spatial and visual-motor coordination, such as replication of an abstract block design or copying a drawing from a model, while visual recognition and matching abilities are relatively unimpaired. Studies using visual psychophysics have reported that adolescents and adults with FXS show reduced contrast sensitivity for low spatial and high temporal frequency visual stimuli known to engage magnocellular pathway processing, but intact sensitivity for high spatial and low temporal frequency stimuli that elicit parvocellular pathway processing (Kogan et al., 2004a). Furthermore, impaired sensitivity for discriminating second-order static and first- and second-order moving visual stimuli, accompanied by near normal sensitivity for first-order static stimuli, has been found in adolescents and adults with FXS (Kogan et al., 2004b). These studies have been seminal in refining the picture of visual deficits in individuals with FXS, suggesting typical ventral stream processing accompanied by either atypical dorsal stream processing or a generalized impairment in higher-level neural mechanisms necessary for integrating dorsal and ventral visual input. These interpretations need not be mutually exclusive. Recent work investigating visual processing in infants with FXS has revealed that a selective deficit in sensitivity for detecting second-order motion stimuli can be identified early in development (Farzin et al., 2008). Because attentive tracking is believed to be necessary for the detection of dynamic secondorder stimuli (Sperling, 1989; Cavanagh, 1992; Johnston et al., 1992; Nishida and Sato, 1995; Seiffert and Cavanagh, 1998), this finding was explained as a deficit in temporal or motion processing in infants with FXS, as either could result in poor attentive tracking and both types of processing are subserved by parietal areas of the brain. These results are consistent with and extend the work of Kogan et al. (2004b) by uncovering the developmental trajectory of the putative dorsal stream deficit in both male and female infants diagnosed with FXS. However, given that infants with FXS performed comparable to TD controls when detecting first-order motion stimuli, which are processed by a passive, velocity sensitive mechanism (Seiffert and Cavanagh, 1998), these results also call into question whether a low-level motion processing or strictly subcortical deficit exists in individuals with FXS. While we cannot directly compare levels of sensitivity obtained from infants in our study to sensitivity found in adults because of differences in stimuli (second-order stimuli are defined differently), tasks (detection versus discrimination), and age groups, we can speculate that FMRP may have a protracted time course in the early development of visual areas.

Other evidence of developmental visual abnormalities comes from studies with toddlers with FXS. First, Scerif et al. (2004) investigated selective visual attention in 2- and 3-year-olds with FXS using a visual search task, and found that the FXS group showed a perseverative error of selecting targets that had previously been found. Another study examined oculomotor control in toddlers with FXS (Scerif- et al., 2005) by presenting children with a task in which the goal was to inhibit saccades toward suddenly appearing peripheral stimuli (prosaccades) and direct them instead to contralateral locations (antisaccades). Consistent with the above finding of deficits in selective visual attention, they found that toddlers with FXS failed to suppress prosaccades toward the cue during the test trials, likely caused by atypical connectivity between parietal and frontal circuitry (McDowell et al., 2008). Taken together, these findings begin to converge on a picture of visual processing in young individuals with FXS in which there exists a disruption in the 'vision-for-action' pathway, or the dorsal stream (Milner and Goodale, 1995).

Here we conducted two experiments to assess how infants with and without FXS process changes in object properties during occlusion events which either involve a dynamic transformation across time and space, engaging dorsal stream processing, or which remain static, engaging ventral stream processing. The tasks were designed based on the hypothesis that dysfunction of parietal areas in processing basic spatial and temporal visual information is likely to be the underpinning source of cognitive impairments found in individuals with FXS. These findings presented here provide us with a better understanding of the development of dynamic object representations in typically and atypically developing infants, bridging the link between specific gene expression, brain development, and cognitive function. 


\section{EXPERIMENT 1 \\ MATERIALS AND METHODS \\ Participants}

Thirty-two infants diagnosed with the FXS full mutation (27 boys and 5 girls) and thirty-four mental age-matched full-term TD infants ( 27 boys and 7 girls) were included in the final sample. Mean chronological age for the FXS and TD groups was 26.37 months $( \pm 7.15$, range $=14-45$ months $)$ and 18.09 months $( \pm 5.51$, range $=11-31$ months), respectively. Data from an additional three TD infants and four infants with FXS were not included in the final analysis because the infant did not provide gaze data on all four test trials. TD infants were recruited through letters to families, fliers, and word of mouth in Davis, California. Infants with FXS were recruited from, and clinically evaluated at, the UC Davis M.I.N.D. Institute Fragile X Research and Treatment Center (FXRTC), and molecular DNA testing was carried out to confirm their diagnosis. The Institutional Review Board at the University of California, Davis, approved the experimental protocol, and informed consent was obtained from a parent or caregiver of each infant.

Cognitive assessment. To control for differences in developmental level, all infants with FXS were assessed using the Mullen Scales of Early Learning (Mullen, 1995) to derive a mental age. The MSEL is a standardized developmental test for children ages 3 to 60 months, consisting of five subscales: gross motor, fine motor, visual reception, expressive language, and receptive language. Infants in the FXS group had a mean mental age of 17.39 months ( \pm 5.76 , range $=11-36$ months), which was matched to infants in the TD group ( 18.35 months \pm 5.35 , range $=11-31$ months $)$. An independent samples t-test confirmed that mental age did not differ significantly between the two final groups $(t(1,64)=0.702$, $p=0.485,2$-tailed).

\section{Apparatus and stimuli}

Stimuli were presented on a Tobii 17 -inch LCD binocular eye tracker $(1024 \times 768$ pixels resolution, $50-\mathrm{Hz}$ capture rate, $60-\mathrm{Hz}$ refresh rate) to record infants' fixations during the task. The calibration procedure was run using ClearView software (Tobii Technology, Sweden), which allows an optimal accuracy of $0.5^{\circ}$.

Stimulus creation and programming were done using Adobe Flash CS4 Professional software. Infants were shown two objects; a red sphere and a green cylinder, each fitting within a 5 deg by 5 deg square, presented 4 deg from the midline, against a black background. Each occluder was a white square that fully covered the object. During the task infants heard audio sequences of classical music through two standard computer speakers concealed behind the monitor.

\section{Procedure}

Infants were seated on a parent or caregiver's lab, $60 \mathrm{~cm}$ from the eye tracker monitor. The experiment began with a five-point calibration routine, followed by a single familiarization trial and four test trials. Figure 1 presents a schematic of the experimental design.

Familiarization trial. A red sphere on the left and a green cylinder on the right were lowered from the top to the middle of the screen at a speed of $3 \mathrm{~cm} / \mathrm{s}$. The objects remained stationary for $5 \mathrm{~s}$, after which they were concealed for $1 \mathrm{~s}$ by the occluders, also lowered from the top of the screen. The occluders were then raised to reveal the objects for $1 \mathrm{~s}$ and were lowered immediately after to re-occlude the shapes for $1 \mathrm{~s}$. The occluders revealed the objects a final time and then dropped back down. The purpose of this trial was to provide infants with sufficient time to visually examine each object and its location, and to learn that the role of the occluders was to hide the objects without transforming them in any way.

\section{Familiarization Trial}
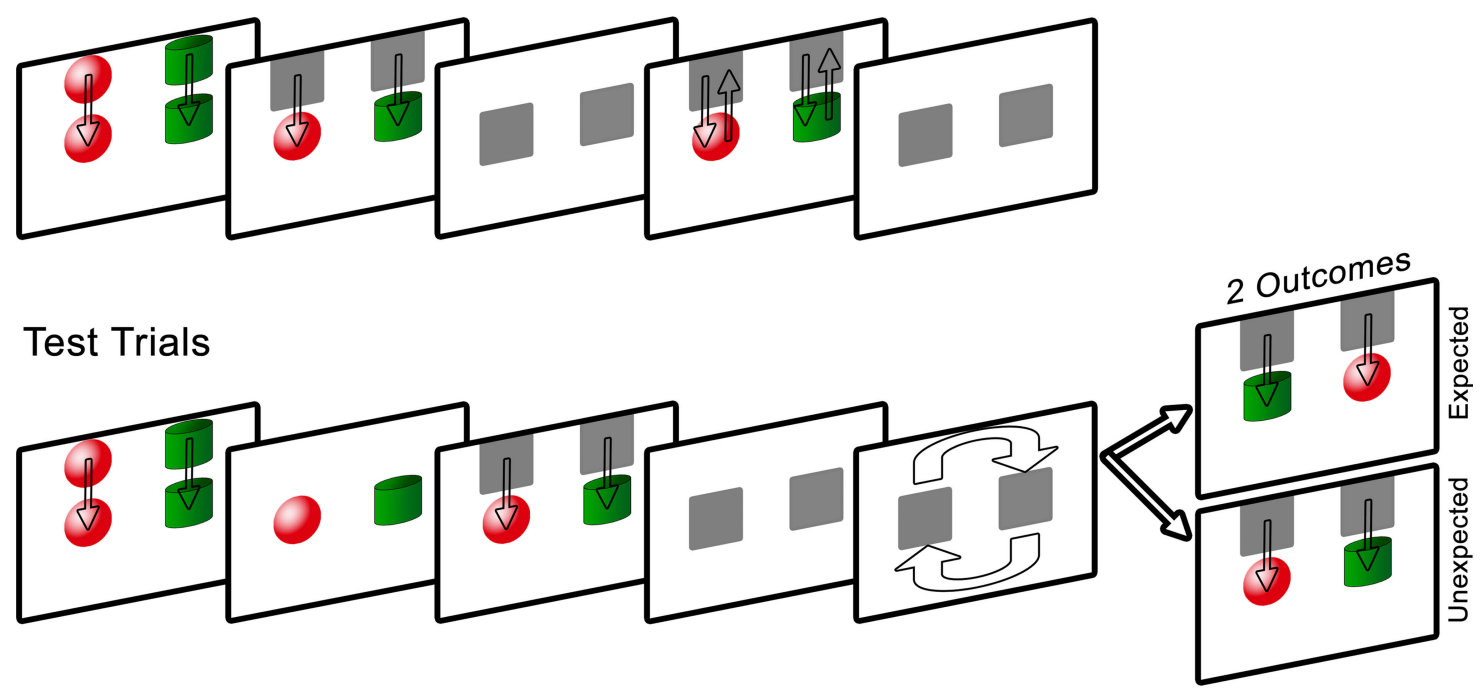

FIGURE 1 | Schematic of Experiment 1 design. Note that on the computer screen the background was black, the occluders were white, and the arrows were not present. 
Test trials. Two pairs of test trials were presented in one of two orders: expected, unexpected, unexpected, expected or unexpected, expected, expected, unexpected, counterbalanced across subjects.

The test trials were similar to the familiarization trial in that following a 5-s viewing period of the objects, the occluders were lowered to cover the objects. During a 5-s occlusion, the occluders rotated $180^{\circ}$ in a clockwise direction (local orientation maintained), after which they were raised to reveal either the objects in their newly rotated positions whereby the cylinder was now on the left (expected outcome) or the objects in their original positions (unexpected outcome). Infants were given $10 \mathrm{~s}$ to view the outcome, after which the occluders were lowered and rotated counterclockwise back to their starting positions, and the screen faded to black. After $2 \mathrm{~s}$ the subsequent test trial began and the sequence repeated until all four test trials were completed. The total task lasted $2 \mathrm{~min}$ and $24 \mathrm{~s}$. It was predicted that if infants successfully encoded and bound the individual object identities to specific spatial locations, and tracked this information during the dynamic occlusion period, they should respond to the violation of object location by looking longer at the unexpected outcome compared to the expected outcome.

\section{Coding}

Eye tracking data was coded using the Area-of-Interest definition tool within ClearView. AOIs were defined by creating a 6 deg by 6 deg square around each object. The primary measure of interest included duration of fixations to each AOI region, where a fixation was defined as a period of looking in which the position of the eyes did not shift more than 30 pixels for a minimum of $200 \mathrm{~ms}$. Fixations outside of the two AOIs were coded as either "away" or off-screen. Coding began once the occluders were raised to reveal half of the objects and ended when the objects were fully re-concealed. Mean looking time during each test trial was then calculated by summing the fixation duration to the two AOIs.

\section{RESULTS AND DISCUSSION}

Preliminary analyses of mean looking time differences between the expected and unexpected outcomes using a repeated measures analysis of variance (ANOVA) showed no effects involving mental age, gender, or trial order; therefore these factors were excluded from the following analyses. Mean looking time during each test trial was entered into a repeated measures ANOVA with two withinsubject factors: outcome (expected or unexpected) and trial pair (first or second), and one between-group factor: group (typically developing or FXS). The analysis revealed a significant main effect of diagnosis $\left(F(1,64)=14.03, p=0.0001, \eta^{2}=0.180\right)$ whereby TD infants looked significantly longer overall $(M=6.31 \mathrm{~s}, S D=2.38 \mathrm{~s})$ compared to infants with FXS $(M=4.29 \mathrm{~s}, S D=2.57 \mathrm{~s})$. A significant interaction between outcome and diagnosis $(F(1$, $64)=7.502, p=0.008, \eta^{2}=0.105$ ) was also found, driven by TD infants' longer looking times during the unexpected $(M=6.86 \mathrm{~s}$, $S D=2.11 \mathrm{~s})$ than the expected $(M=5.76 \mathrm{~s}, S D=2.55 \mathrm{~s})$ outcome test trials $(t(32)=2.556, p=0.015, \mathrm{SEM}=0.43)$. Infants with FXS trended toward longer looking during the expected $(M=4.52 \mathrm{~s}$, $S D=2.67 \mathrm{~s})$ than the unexpected $(M=4.05 \mathrm{~s}, S D=2.47 \mathrm{~s})$ outcome test trials $(t(31)=-1.507, p=0.142$, SEM $=0.35)$. A binomial sign confirmed that while 24 out of $34(71 \%, p=0.024) \mathrm{TD}$ infants looked longer at the unexpected compared to expected outcome trials, only 16 out of $32(50 \%, p=1.14)$ infants with FXS looked longer at unexpected outcome trials. No other significant effects or interactions were found. Figure 2 shows mean looking times during the expected and unexpected test trial outcomes for the two groups.

Perhaps infants with FXS neglected to track the movement of the occluders during the rotation period, thereby leaving them unable to respond to the unexpected object location. To answer this question, a third AOI was created which encompassed the area through which the occluders moved, and looking time was calculated for each infant and each test trial. The result of an independent samples t-test confirmed that mean looking time to the occluders during the rotation periods did not differ significantly between the two groups $(t(1,64)=2.255, p=0.136,2$-tailed $)$, thus the failure of infants with FXS to look longer at the unexpected outcome cannot be attributed to differences in looking time to the rotation of the occluders. There was also no difference in looking time during the familiarization period between infants with and without FXS $(t(1,64)=2.920, p=0.092,2$-tailed $)$, which could have influenced perceptual novelty preferences.

These results demonstrate that TD infants successfully maintained the identity of an occluded object across a spatiotemporal transformation, and infants with FXS did not. Instead, infants with FXS showed a tendency to look longer during the expected test trial outcome, suggesting that the object representation was not maintained throughout the rotation of the occluders. Thus, objects in their new positions may have violated the expectation that object features would remain bound to their original locations. This looking pattern was not dependent on age, sex, test trial order, looking time during the familiarization trial, or time spent looking at the occluders during the transformation period.

Attentive tracking of occluded objects is believed to involve correspondence matching of features across changing spatial positions over time, a function shown to be mediated by parietal and frontal areas of the brain (Cavanagh, 1992; Yantis, 1992; Culham et al., 1998, 2001). Therefore, these results support the hypothesis that infants with FXS are impaired on an object occlusion task

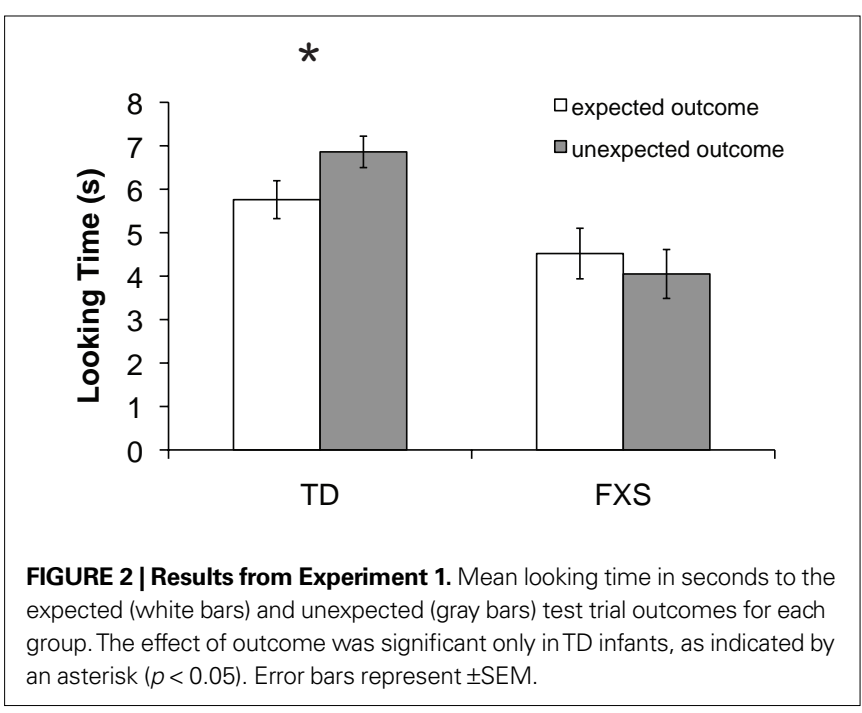


requiring dorsal stream function, either as a result of a selective spatial or temporal processing deficit or a deficit in the processing of integrated spatiotemporal information.

\section{EXPERIMENT 2}

To rule out the possibility that infants with FXS were unable to maintain an object representation during a period of static occlusion, a control experiment was performed using the same experimental procedure but removing the dynamic quality of the occlusion. Namely, in this experiment, there was no spatiotemporal transformation during the occlusion period; rather, the occluders remained static and the object property that changed during the occlusion period was color. Color was selected as the feature violation because it is known to differentially activate the ventral pathway. The question was whether infants with FXS would be able to form accurate expectations about the identity of the occluded objects in the absence of the occluders' motion.

\section{MATERIALS AND METHODS}

The method was the same as in Experiment 1 unless otherwise noted.

\section{Participants}

Twenty-four infants diagnosed with the FXS full mutation (19 boys and 4 girls) and thirty-three mental age-matched full-term TD infants ( 25 boys and 8 girls) were included in the final sample. Mean chronological age for the FXS and TD groups was 27.52 months $( \pm 8.36$, range $=11-46$ months $)$ and 16.52 months
( \pm 5.15 , range $=11-32$ months), respectively. Infants in the FXS group had a mean mental age of 16.54 months $( \pm 5.74$, range $=11-28$ months), matched to infants in the TD group (16.88 months \pm 4.96 , range $=11-32$ months $)$. There was no significant difference between the two groups on developmental level $(t(1,55)=0.244, p=0.809,2$-tailed $)$.

Twelve TD infants were not included as a result of poor calibration (1), parent verbal interference (1), or incomplete gaze data for all four test trials (10). Data from an additional eight infants with FXS were not included in the final analysis because of poor calibration (2) or incomplete gaze data (6).

\section{Apparatus and stimuli}

Infants were shown two objects; an orange triangle and a blue circle, each fitting within a $5 \mathrm{deg}$ by $5 \mathrm{deg}$ square region, presented $4 \mathrm{deg}$ from the midline, against a black background. Each occluder was a white square that fully covered the object.

\section{Procedure}

Figure 3 presents a schematic of the experimental design.

Familiarization trial. Infants were presented with a blue triangle on the left and an orange circle on the right, lowered from the top to the middle of the screen at a speed of $3 \mathrm{~cm} / \mathrm{s}$. The objects remained stationary for $5 \mathrm{~s}$, after which the occluders were lowered to conceal the objects for $1 \mathrm{~s}$. The occluders were then raised to reveal the objects for $1 \mathrm{~s}$ and were immediately lowered to re-occlude the shapes for $1 \mathrm{~s}$. This process was repeated a second time.

\section{Familiarization Trial}

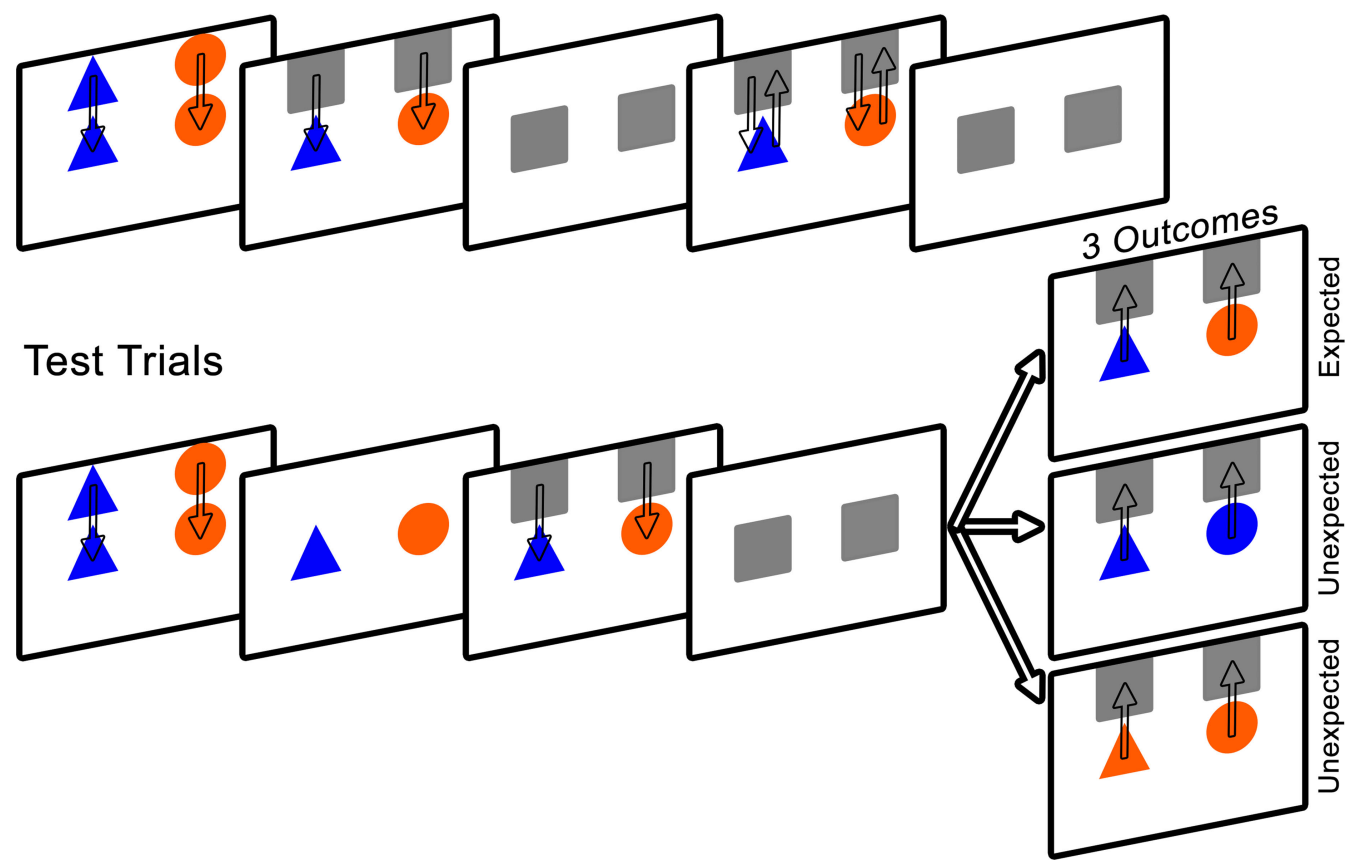

FIGURE 3 | Schematic of Experiment 2 design. Note that on the computer screen the background was black, the occluders were white, and the arrows were not present. 
Test trials. Two pairs of test trials were presented in one of two orders: expected, unexpected, unexpected, expected or unexpected, expected, expected, unexpected, counterbalanced across infants.

The test trials were similar to the familiarization trial in that the objects were presented and remained stationary for $5 \mathrm{~s}$. The occluders were lowered to cover the objects for a period of $5 \mathrm{~s}$, during which they remained stationary.

The occluders were then raised to reveal either the unchanged objects (expected outcome) or one object that had changed color (unexpected outcome). To avoid presenting infants with a novel color, the object was changed to match the color of the second object (orange to blue or blue to orange; color of changed object alternated on unexpected trials). Infants were given $10 \mathrm{~s}$ to view the trial outcome, after which the occluders were lowered and the screen faded to black. After $2 \mathrm{~s}$, the subsequent test trial began. The total task lasted $2 \mathrm{~min}$ and $24 \mathrm{~s}$. We expected that if infants encoded and bound the features of each object to their appropriate spatial location, and retained this information during the static occlusion, they should respond to the violation of color by demonstrating increased looking time to the unexpected outcome compared to the expected outcome trials. More specifically, infants should look longer at the spatial location of the object whose identity changed.

\section{Coding}

Coding was the same as in Experiment 1. In addition to mean looking time during each test trial, a Visual Preference (VP) score, indexing the proportion of looking time to the object that changed color, was calculated using the following formula: (looking time to changed object)/(looking time to both objects). VP scores could be between 0 and 1 , with 0.5 considered equal preference for both objects. For each infant, a VP score was calculated for each unexpected outcome test trial ${ }^{1}$.

\section{RESULTS AND DISCUSSION}

Preliminary analyses of mean looking time differences between the expected and unexpected outcomes using a repeated measures ANOVA showed no significant effects involving mental age, gender, or trial order; therefore these factors were excluded from further analyses. Mean looking time during each test trial was entered into a repeated measures ANOVA with two within-subject factors: outcome (expected or unexpected) and trial pair (first or second), and one between-group factor: group (typically developing or FXS). The analysis revealed a significant main effect of outcome $\left(F(1,55)=13.39, p=0.001, \eta^{2}=0.196\right)$ in which infants looked longer at the unexpected $(M=5.32 \mathrm{~s}, S D=2.98 \mathrm{~s})$ compared to the expected $(M=3.19, S D=2.12 \mathrm{~s})$ outcome test trials. A significant main effect of trial pair $\left(F(1,55)=6.44, p=0.014, \eta^{2}=0.105\right)$ was also found, resulting from longer looking times during the first trial pair $(M=5.22 \mathrm{~s}, S D=3.33 \mathrm{~s})$ than the second $(M=4.34 \mathrm{~s}$,

${ }^{1}$ Note that a VP score calculation was not possible for Experiment 1 because the unexpected outcome consisted of both objects in their original positions and there was therefore no reason to believe that infants would choose to look at the location of one object longer than the other.
$S D=3.24 \mathrm{~s}$ ), most likely an effect of boredom or fatigue toward the end of the task. No other significant effects or interactions were found. Critically, there was not a significant interaction between outcome and group $(F(1,55)=0.572, p=0.453)$. Figure 4 shows mean looking times to the expected and unexpected test trial outcomes for the two groups.

These findings were further explored by conducting a series of planned comparisons (paired t-tests) showing that TD infants looked significantly longer to the unexpected $(M=6.44 \mathrm{~s}$, $S D=3.15 \mathrm{~s})$ than the expected $(M=5.55 \mathrm{~s}, S D=3.04 \mathrm{~s})$ outcome test trial for the first pair of test trials $(t(32)=2.441$, $p=0.019, \mathrm{SEM}=0.59)$ and significantly longer to the unexpected $(M=5.69 \mathrm{~s}, S D=3.28 \mathrm{~s})$ than the expected $(M=4.06 \mathrm{~s}$, $S D=2.56 \mathrm{~s})$ outcome test trial for the second pair of test trials $(t(32)=3.216, p=0.003, \mathrm{SEM}=0.50)$. Infants with FXS looked significantly longer to the unexpected $(M=4.65 \mathrm{~s}$, $S D=3.11 \mathrm{~s})$ than the expected $(M=3.54 \mathrm{~s}, S D=3.55 \mathrm{~s})$ outcome test trial for the first pair of test trials $(t(23)=2.514$, $p=0.020, \mathrm{SEM}=0.43$ ) and trended toward longer looking to the unexpected $(M=3.88 \mathrm{~s}, S D=3.47 \mathrm{~s})$ compared to expected outcome $(M=3.28 \mathrm{~s}, S D=3.39 \mathrm{~s})$ test trial for the second pair

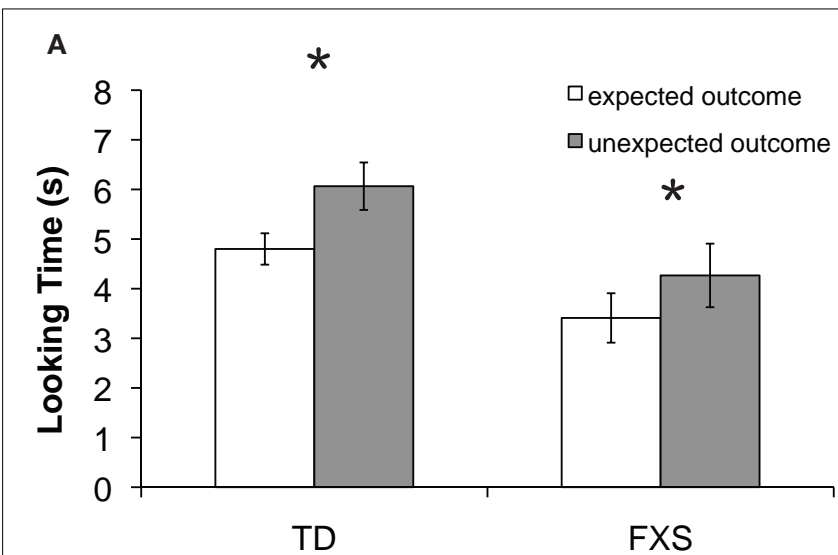

B

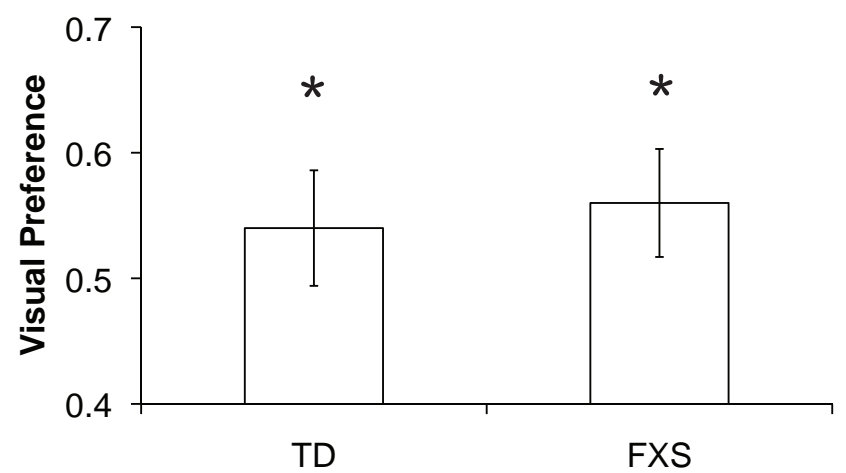

FIGURE 4 | Results from Experiment 2. (A) Mean looking time in seconds to the expected (white bars) and unexpected (gray bars) test trial outcomes for each group. The effect of outcome was significant in both groups as indicated by an asterisk $(p<0.05)$. (B) Mean visual preference for the changed object for each group. Asterisks indicate significant difference from chance $(p<0.05)$. Error bars represent \pm SEM. 
of test trials $(t(23)=0.956, p=0.349$, SEM $=0.62)$. A binomial sign test was carried out separately for each group of infants, and confirmed that 18 out of $24(75 \%, p=0.023)$ infants with FXS looked longer during unexpected outcome test trials, while 27 out of 33 (82\%, $p=0.0001)$ TD infants looked longer during unexpected compared to expected outcome test trials.

No group difference was identified for VP for the changed object during the unexpected outcome trials $[F(1,56)=1.698$, $p=0.199$ ], and overall scores were significantly greater than the chance level of $0.5(t(55)=2.375, p=0.021, \mathrm{SEM}=0.035)$, indicating reliable discrimination of the changed object identity (Figure 4).

The results from Experiment 2 indicate that during an occlusion event in which objects remained static, thereby minimizing dorsal stream involvement in motion tracking, TD infants and infants with FXS were able to maintain object representations such that a change in color of one of the objects elicited a violation of the infant's visual expectation. Further, during unexpected outcome test trials, infants in both groups spent a significantly greater proportion of time looking specifically at the location of the object that underwent the color change. Most importantly, this looking pattern was found independent of diagnosis, ruling out the possibility that infants with FXS have difficulty in encoding and maintaining the identity-location binding of an occluded object or exhibit a generalized working memory deficit. While we acknowledge that object properties such as color or motion are unlikely to elicit purely ventral or dorsal representations, because color is mostly restricted to ventral-stream processing, the results of Experiment 2 lead to the conclusion that infants with FXS have relatively spared ventralstream object processing.

Results from this experiment replicate those of previous work showing that TD infants are capable of mentally representing the featural identity of a static object during a period of occlusion, and that there is differential processing of stimulus features such that by 12 -months infants can use color information when reasoning about objects.

\section{GENERAL DISCUSSION}

The experiments reported here were designed to investigate object processing of dynamic and static occlusion events in infants with and without FXS as a means of explicitly testing dorsal and ventral visual abilities. This is the first study to experimentally examine object processing skills in infants with FXS. Our findings reveal that, in contrast to mental age-matched TD infants, infants with FXS were unable to mentally represent and track the identity of occluded objects during a dynamic event. However, infants with FXS performed comparable to controls when representing the identity of objects during a static occlusion event. Thus, infants with FXS appear to have a selective deficit in the visual processing required for object tracking, supporting the hypothesis of a deficit of dorsal stream functioning. While these results are consistent with a selective dorsal stream deficit, an equally plausible explanation is that infants with FXS may have difficulty in maintaining integrated ventral and dorsal information during a dynamic occlusion event. Both interpretations involve the reduced ability to represent the spatial position of a moving object, which is subserved by higher stages of visual processing including but not limited to parietal cortical areas of the brain (Culham et al., 1998; Battelli et al., 2001, 2007).

The mechanisms involved in maintaining the representation of an occluded object across changes in time and space are thought to primarily involve selective visual attention (Awh and Jonides, 1999; Scholl et al., 2001) and visual working memory (Pasternak and Greenlee, 2005; Xu and Chun, 2006). Several functional neuroimaging studies have shown that increased response of posterior parietal cortex is associated with maintaining spatiotemporal information about an object in working memory (Olson et al., 2003; Todd and Marois, 2004; Shuwairi et al., 2007). This is thought to be part of a larger cortical network involved in perceptual completion, motion perception, and timing estimation. Further, in adults, the prefrontal cortex has been found to play a critical role in integrating dorsal and ventral stream information required for action planning and execution by the dorsal stream as well as for maintaining visual information in working memory (Rao et al., 1997; Rossetti and Pisella, 2002; Mohr and Linden, 2005).

These data add to the growing body of research suggesting that a dorsal stream deficit is present in individuals with FXS, and extend the existing findings by showing that this deficit is present during infancy. These results are supported by evidence from children and adults with FXS demonstrating lower performance on other tasks mediated by the dorsal stream such as coherent motion processing, visual-motor coordination, and basic numerical computation (Cornish et al., 1999; Rivera et al., 2002; Kogan et al., 2004). Additional studies that disentangle the spatial from the temporal aspects of visual processing are required in order to fully understand the observed object tracking impairment in infants with FXS.

\section{CONCLUSION}

Our visual system encounters the constant challenge of encoding, maintaining, and tracking object information in our dynamic environment. Here we show that TD infants can reliably represent and track an occluded object across changes in time and space, while infants diagnosed with FXS cannot. These findings provide important baseline data from which to understand the developmental trajectory of visual and cognitive processing in both groups of infants. We conclude that infants with FXS experience disrupted dorsal stream processing, known to be directly involved in spatiotemporal tracking of objects. It is not clear how early in development this visual processing impairment may arise or if there is a critical period for the involvement of FMRP in the early development this network of areas. Future experiments are needed to further examine the nature of this spatial and/or temporal visual processing deficit, particularly as it relates to downstream cognitive abilities characteristic of the disorder.

\section{ACKNOWLEDGMENTS}

The authors thank David Whitney for helpful discussions, and the infants and their caregivers for participating. A portion of these data was presented at the SSBP annual meeting in October 2007. This work was supported by NIH grants R01HD056031 (Susan M. Rivera) and F31MH083386 (Faraz Farzin). 


\section{REFERENCES}

Atkinson, J. (1993). A neurobiological approach to the development of "where" and "what" systems for spatial representation in human infants. In Spatial Representation: Problems in Philosophy and Psychology, N. Eilan, R. McCarthy and B. Brewer, eds (Oxford, UK, Blackwell), pp. 325-339.

Atkinson, J. (2000). The Developing Visual Brain. Oxford, Oxford University Press.

Awh, E., and Jonides, J. (1999). Overlapping mechanisms of attention and spatial working memory. Trends Cogn. Sci. (Regul. Ed.) 10, 433-437.

Baillargeon, R. (1993). The object concept revisited: new directions in the investigation of infants' physical knowledge. In Visual Perception and Cognition in Infancy, C. E. Granrud, ed. (London, Lawrence Erlbaum Associates), pp. 265-315.

Battelli, L., Cavanagh, P., Intriligator, J., Tramo, M. J., Henaff, M. A., Michel, F., and Barton, J. J. (2001). Unilateral right parietal damage leads to bilateral deficit for high-level motion. Neuron 32, 985-995.

Battelli, L., Pascual-Leone, A., and Cavanagh, P. (2007). The 'when' pathway of the right parietal lobe. Trends Cogn. Sci. (Regul. Ed.) 11, 204-210.

Beckett, L., Qilu, Y., and Long, A. N. (2005). The Impact of Fragile X: Prevalence, Numbers Affected, and Economic Impact. A white paper prepared for the National Fragile X Foundation. Davis, CA, University of California.

Brown, W. T., Jenkins, E. C., Friedman, E., Brooks, J., Wisniewski, K., Raguthu, S., and French, J. (1982). Autism is associated with the fragile-X syndrome. J. Autism Dev. Disord. 12, 303-308.

Cavanagh, P. (1992). Attention-based motion perception. Science 257, 1563-1565.

Comery, T. A., Harris, J. B., Willems, P. J., Oostra, B. A., Irwin, S. A., Weiler, I. J., and Greenough, W. T. (1997). Abnormal dendritic spines in fragile $\mathrm{X}$ knockout mice: maturation and pruning deficits. Proc. Natl. Acad. Sci. U.S.A. 94, 5401-5404.

Cornish, K. M., Munir, F., and Cross, G. (1999). Spatial cognition in males with Fragile-X syndrome: evidence for a neuropsychological phenotype. Cortex 35, 263-271.

Crawford, D., Acuna, J. M., and Sherman, S. L. (2001). FMR1 and the fragile X syndrome: human genome epidemiology review. Genet. Med. 3, 359-371.

Culham, J. C., Brandt, S. A., Cavanagh, P., Kanwisher, N. G., Dale, A. M., and Tootell, B. (1998). Cortical fMRI activation produced by attentive tracking of moving targets. J. Neurophysiol. 80, 2657-2670.

Culham,J.C., Cavanagh, P., and Kanwisher, N.G. (2001).Attention response functions: Characterizing brain areas using fMRI activation during parametric variations of attentional load. Neuron 32, 737-745.

Farzin, F., Whitney, D., Hagerman, R. J., and Rivera, S. M. (2008). Contrast detection in infants with fragile $\mathrm{X}$ syndrome. Vision Research, 48, 1471-1478.

Irwin, S. A., Galvez, R., Weiler, I. J., BeckelMitchener, A., and Greenough, W. T. (2002). Brain structure and functions of fMR1 protein. In Fragile X Syndrome: Diagnosis, Treatment and Research, 3rd Edn, R.J.Hagerman and P.J.Hagerman, eds (Baltimore, The Johns Hopkins University Press), pp. 191-205.

Johnson, M. H., Mareschal, D., and Csibra, G. (2001). The functional development and integration of the dorsal and ventral visual pathways: a neurocomputational approach. In The Handbook of Developmental Cognitive Neuroscience, C. A. Nelson and M. Luciana eds (Cambridge, MA, MIT Press), pp. 339-351.

Johnston, A., McOwan, P.W., and Buxton, H. (1992). A computational model of the analysis of some first-order and second-order motion patterns by simple and complex cells. Proc. R. Soc. Lond. B. 250, 297-306.

Kaldy, Z., and Leslie, A., M. (2003) Identification of objects in 9 month old infants: integrating what and where information. Dev. Sci. 6, 360-373.

Kogan, C. S., Boutet, I., Cornish, K., Zangenehpour, S., Mullen, K. T., Holden, J. J., Der Kaloustian, V. M., Andermann, E., and Chaudhuri, A (2004a). Differential impact of the fMR1 gene on visual processing in fragile X syndrome. Brain 127(Pt. 3), 591-601.

Kogan, C. S., Bertone, A., Cornish, K., Boutet, I., Der Kaloustian, V. M., Andermann, E., Faubert, J., and Chaudhuri, A. (2004b). Integrative cortical dysfunction and pervasive motion perception deficit in fragile $\mathrm{X}$ syndrome. Neurology 63, 1634-1639.

Leslie, A. M., and Kaldy, Z. (2001). Indexing individual objects in infant working memory. J. Exp. Child. Psychol. 78, 61-74.
Leslie, A. M., Xu, F., Tremoulet, P. D., and Scholl, B. J. (1998). Indexing and the object concept: developing 'what' and 'where' systems. Trends Cogn. Sci. (Regul. Ed.) 2, 10-18.

Mareschal, D., and Johnson, M.H. (2003) The "what" and "where" of object representations in infancy. Cognition 88, 259-276a.

McDowell, J. E., Dyckman, K. A., Austin B. P., and Clementz, B. A. (2008). Neurophysiology and neuroanatomy of reflexive and volitional saccades: evidence from studies of humans. Brain Cogn. 68, 255-270.

Milner, A. D., and Goodale, M. A. (1995) The Visual Brain in Action. Oxford Oxford University Press.

Mohr, H. M., and Linden, D. E. J. (2005) Separation of the systems for color and spatial manipulation in working memory revealed by a dual-task procedure. J. Cogn. Neurosci. 17, 355-366.

Mullen, E. M. (1995). Mullen Scales of Early Learning. Circle Pines, MN, American Guidance Service Inc.

Nishida, S., and Sato, T. (1995). Motion aftereffect with flickering test patterns reveal higher stages of motion processing. Vision Res. 35, 477-490.

Olson, I. R., Gatenby, J. C., Leung, H. C., Skudlarski, P., and Gore, J. C. (2003). Neuronal representation of occluded objects in the human brain. Neuropsychologia 42, 95-104.

Pasternak, T., and Greenlee, M. (2005) Working memory in primate visual systems. Nat. Rev. Neurosci. 6, 97-107.

Rao, S. C., Rainer, G., and Miller, E. K. (1997). Integration of what and where in the primate prefontal cortex. Science 276, 821-824.

Reddy, K. S. (2005). Cytogenetic abnormalities and fragile-X syndrome in Autism Spectrum Disorder. BMC Med. Genet. 6, 3 .

Richardson, D. C., and Kirkham, N. Z (2004). Multimodal events and moving locations: Eye movements of 6 month-olds reveal dynamic spatial indexing. J. Exp. Psychol. Gen. 133 46-62.

Rivera, S. M., Menon, V., White, C. D. Glaser, B., and Reiss, A. L. (2002). Functional brain activation during arithmetic processing in females with fragile X Syndrome is related to FMR1 protein expression. Hum. Brain Mapp. 16, 206-18.

Rossetti, Y., and Pisella, L. (2002). Several "vision for action" systems: A guide to dissociating and integrating dorsal and ventral functions. In Attention and Performance
XIX: Common mechanisms in Perception and Action. W. Prinz and B. Hommel, eds (Oxford, England, Oxford University Press). pp. 62-119.

Scerif, G., Cornish, K., Wilding, J., Driver, J., and Karmiloff-Smith, A. (2004). Visual search in typically developing toddlers and toddlers with Fragile X or Williams syndrome. Dev. Sci. 7, 116-130.

Scerif, G., Karmiloff-Smith, A., Campos, R., Elsabbagh, M., Driver, J., and Cornish, K. (2005). To look or not to look? Typical and atypical development of oculomotor control. J. Cogn. Neurosci. 17, 591-604.

Scholl, B. J., Pylyshyn, Z. W., and Feldman, J. (2001). What is a visual object? Evidence from target merging in multiple object tracking. Cognition 80, 159-177.

Seiffert, A. E., and Cavanagh, P. (1998). Position displacement, not velocity, is the cue to motion detection of second-order stimuli. Vision Res. 38, 3569-3582.

Shuwairi, S. M., Curtis, C. E., and Johnson, S. P. (2007). Neural substrates of dynamic object occlusion. J. Cogn. Neurosci. 19, 1275-1285.

Spelke, S. E., Katz, G., Purcell, S.E., Ehrlich, S. M., and Breilinger, K. (1994). Early knowledge of object motion: continuity and inertia. Cognition 51, 131-176.

Sperling, G. (1989). Three stages and two systems of visual processing. Spat. Vis. 4, 183-207.

Todd, J. J., and Marois, R. (2004). Capacity limit of visual short-term memory in human posterior parietal cortex. Nature 428, 751-754.

Tremoulet, P. D., Leslie, A. M., and Hall, D. G. (2000). Infant individuation and identification of objects. Cogn. Dev. 15, 499-522.

Ungerleider, L. G., and Mishkin, M. (1982). Two cortical visual systems. In Analysis of Visual Behavior, D. J. Ingle, M. A. Goodale and R. J. W. Mansfield, eds (Cambridge, MIT Press). pp. 549-586.

Verkerk, A. J., Pieretti, M., Sutcliffe, J. S., Fu, Y.H., Kuhl, D. P., Pizzuti, A., Reiner, O., Richards, S., Victoria, M.F., Zhang, F. P., Eussen, B.E., van Ommen, G.J. B., Blonden, L. A. J., Riggins, G. J., Chastain, J. L., Kunst, C. B., Galjaard, H., Caskey, C. T., Nelson, D. L. Oostra, B.A., and Warren, S. T. (1991). Identification of a gene (FMR-1) containing a CGG repeat coincident with a breakpoint cluster region exhibiting length variation in fragile $\mathrm{X}$ syndrome. Cell 65, 905-914. 
Wilcox, T. (1999). Object individuation: infants' use of shape, size, pattern, and color. Cognition 72, 125-166.

Wilcox, T., and Schweinle, A. (2002). Object individuation and event mapping: developmental changes in infants' use of featural information. Dev. Sci. 5, 132-150.

Xu,Y., and Chun,M.M. (2006).Dissociable neural mechanisms supporting visual short-term memory for objects. Nature440, 91-95.

Yantis, S. (1992). Multi-element visual tracking: attention and perceptual organization. Cogn. Psychol. 24, 295-340.

Conflict of Interest Statement: The authors declare that the research was conducted in the absence of any commercial or financial relationships that could be construed as a potential conflict of interest.

Received: 02 September 2009; paper pending published: 23 October 2009; accepted: 03 February 2010; published online: 02 March 2010.

Citation: Farzin F and Rivera SM (2010) Dynamic object representations in infants with and without fragile $X$ syndrome. Front. Hum. Neurosci. 4:12. doi: 10.3389/neuro.09.012.2010

Copyright $($ C) 2010 Farzin and Rivera. This is an open-access article subject to an exclusive license agreement between the authors and the Frontiers Research Foundation, which permits unrestricted use, distribution, and reproduction in any medium, provided the original authors and source are credited. 